The Astrophysical Journal, 653:152-158, 2006 December 10

(C) 2006. The American Astronomical Society. All rights reserved. Printed in U.S.A.

\title{
THE MASS OF THE BLACK HOLE IN THE SEYFERT 1 GALAXY NGC 4593 FROM REVERBERATION MAPPING
}

\author{
Kelly D. Denney, ${ }^{1}$ Misty C. Bentz, ${ }^{1}$ Bradley M. Peterson, ${ }^{1}$ Richard W. Pogge, ${ }^{1}$ Edward M. Cackett ${ }^{2}$ \\ Matthias Dietrich, ${ }^{1}$ Jeffrey K. J. Fogel ${ }^{3}$ Himel Ghosh, ${ }^{1}$ Keith D. Horne, ${ }^{2}$ Charles Kuehn, ${ }^{1,4}$ \\ Takeo Minezaki, ${ }^{5}$ Christopher A. Onken, ${ }^{1,6}$ Vladimir I. Pronik, ${ }^{7,8}$ Douglas O. Richstone, ${ }^{3}$ \\ Sergey G. Sergeev, ${ }^{7,8}$ Marianne Vestergaard, ${ }^{9}$ Matthew G. Walker, ${ }^{3}$ and Yuzuru YoshiI ${ }^{5,10}$ \\ Received 2006 July 20; accepted 2006 August 18
}

\begin{abstract}
We present new observations leading to an improved black hole mass estimate for the Seyfert 1 galaxy NGC 4593 as part of a reverberation-mapping campaign conducted at the MDM Observatory. Cross-correlation analysis of the $\mathrm{H} \beta$ emission-line light curve with the optical continuum light curve reveals an emission-line time delay of $\tau_{\text {cent }}=$ $3.73 \pm 0.75$ days. By combining this time delay with the $\mathrm{H} \beta$ line width, we derive a central black hole mass of $M_{\mathrm{BH}}=(9.8 \pm 2.1) \times 10^{6} M_{\odot}$, an improvement in precision of a factor of several over past results.

Subject headings: galaxies: active — galaxies: nuclei — galaxies: Seyfert
\end{abstract}

\section{INTRODUCTION}

Over the past two decades, reverberation mapping (Blandford \& McKee 1982; Peterson 1993) has emerged as the most widely applicable method for directly measuring the masses of supermassive black holes (SMBHs) in active galactic nuclei (AGNs). The appeal of this method arises from its physical simplicity, despite the complicated physics within the central regions of these galaxies. Moreover, unlike other methods for measuring the masses of SMBHs, it does not require high angular resolution. On the other hand, this method is challenging because it requires a large series of observations, well spaced in time and taken over a long period (at least 3 times the length of the longest physical timescale of interest).

The reverberation-mapping method depends on continuum flux variations from the central source, presumably an accretion disk around a SMBH. Continuum photons are absorbed and then reemitted as line photons in the broad-line-emitting region (BLR). Variations in the broad-line flux have a time lag relative to the continuum, corresponding to the light-travel time across the BLR. Through measurements of this time lag, the size of the region

\footnotetext{
1 Department of Astronomy, Ohio State University, 140 West 18th Avenue, Columbus, OH 43210; denney@astronomy.ohio-state.edu, bentz@astronomy .ohio-state.edu, dietrich@astronomy.ohio-state.edu, ghosh@astronomy.ohio-state .edu, peterson@astronomy.ohio-state.edu,pogge@astronomy.ohio-state.edu.

2 School of Physics and Astronomy, University of St. Andrews, Fife, KY16 9SS, Scotland, UK; emc14@st-and.ac.uk, kdh1@st-and.ac.uk.

3 Department of Astronomy, University of Michigan, Ann Arbor, MI 481091090; fogel@umich.edu,dor@umich.edu,mgwalker@umich.edu.

4 Current address: Department of Physics and Astronomy, 3270 Biomedical Physical Sciences Building, Michigan State University, East Lansing, MI 48824; kuehncha@msu.edu.

5 Institute of Astronomy, School of Science, University of Tokyo, 2-21-1 Osawa, Mitaka, Tokyo 181-0015, Japan; minezaki@ioa.s.u-tokyo.ac.jp,yoshii@ ioa.s.u-tokyo.ac.jp.

${ }^{6}$ Current address: National Research Council Canada, Herzberg Institute of Astrophysics, 5071 West Saanich Road, Victoria, BC V9E 2E, Canada; christopher .onken@nrc-cnrc.gc.ca.

7 Crimean Astrophysical Observatory, Nauchny Post Office, 98409 Crimea, Ukraine; sergeev@crao.crimea.ua, vpronik@crao.crimea.ua.

8 Isaak Newton Institute of Chile, Crimean Branch, Ukraine.

9 Steward Observatory, University of Arizona, 933 North Cherry Avenue, Tucson, AZ 85721; mvestergaard@as.arizona.edu.

${ }_{10}$ Research Center for the Early Universe, School of Science, University of Tokyo, 7-3-1 Hongo, Bunkyo-ku, Tokyo 113-0033, Japan.
}

can be determined, which then leads, through virial arguments and a measurement of the broad-line width, to the black hole mass, $M_{\mathrm{BH}}$.

To date, black hole masses have been determined for nearly 40 AGNs using reverberation mapping (see the compilation by Peterson et al. 2004). While this demonstrates how far the field has progressed, Peterson et al. (2004) make it clear that substantial work is still needed to decrease uncertainties in time lag, and thus $M_{\mathrm{BH}}$ measurements, in order to better test and calibrate the various black hole mass-dependent scaling relationships, such as the $M_{\mathrm{BH}}-\sigma_{\star}$ relationship, where $\sigma_{\star}$ is the bulge velocity dispersion. This need for better reverberation-based mass estimates is the driving force behind the campaign we describe below.

One requirement for a successful reverberation-mapping program is good temporal sampling (Horne et al. 2004). The major goal of the current observing program is to improve the sampling rate for reverberation-mapped AGNs that were undersampled in previous campaigns. The mass of the black hole in NGC 4593 was previously measured by reverberation mapping (Dietrich et al. 1994; Onken et al. 2003), but the $\mathrm{H} \beta$ lag determination of $\tau_{\text {cent }}=3.1$ days was unfortunately much smaller than the average interval between observations of 15.8 days. This resulted in uncertainties $(+7.1,-5.1$ days $)$ much larger than the time delay itself, and as such, was consistent with no lag at all. By revisiting this object with our program, we were able to achieve the sampling necessary to drive down the previous high uncertainties in the time lag and black hole mass. Here we present new lag and $M_{\mathrm{BH}}$ determinations from reverberation mapping of NGC 4593. The observational uncertainties from this campaign represent a factor-of-several improvement over previous results.

\section{OBSERVATIONS AND DATA ANALYSIS}

Observations of NGC 4593 were obtained as part of a large reverberation-mapping campaign undertaken in early 2005 on the $1.3 \mathrm{~m} \mathrm{McGraw}-\mathrm{Hill}$ telescope of the MDM Observatory on Kitt Peak in Arizona. Details beyond the descriptions below, for our spectral and photometric observations and data reduction, are given by Bentz et al. (2006b). NGC 4593 is one of three AGNs from which we were able to observe sufficient variability within the time frame of our program to warrant a reverberation analysis. It was first recognized as a host to a type 1 AGN by Lewis et al. (1978), as part of the Michigan-Tololo Curtis Schmidt survey for 


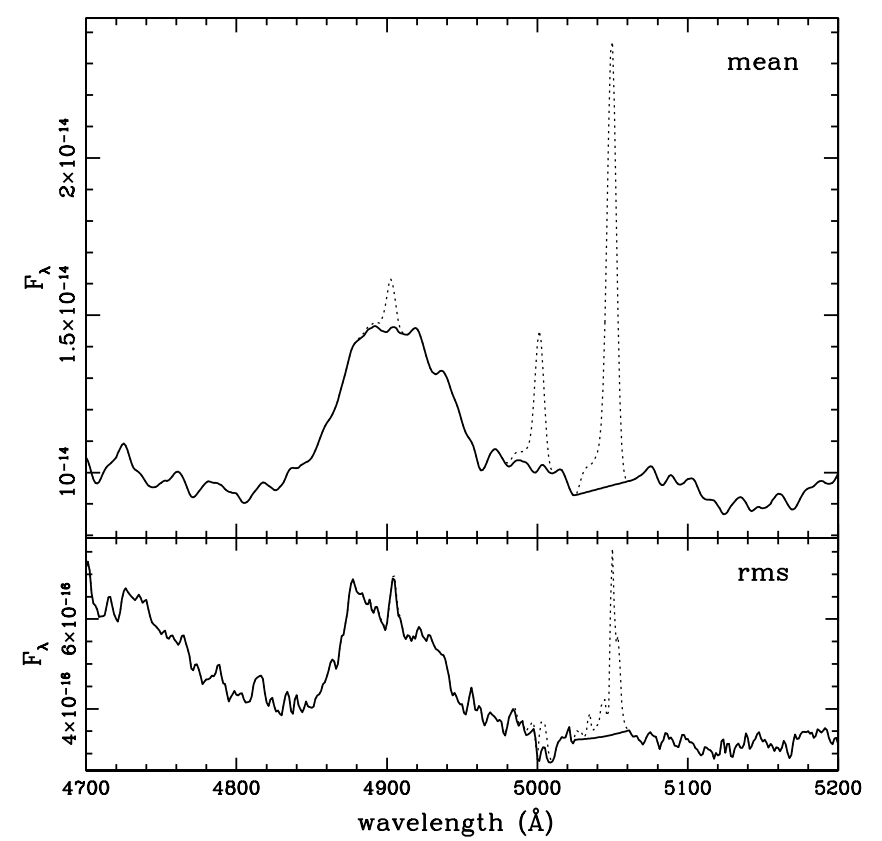

FIG. 1.-Mean and rms spectrum of NGC 4593 from MDM observations. The solid line shows the spectrum with the narrow-line components of $\mathrm{H} \beta$, [O III] $\lambda 4959$, and [O III] $\lambda 5007$ removed. The dotted line shows where these narrow-line components contributed to the spectrum before they were removed. The large increase in rms flux shortward of $4800 \AA$ is due to variations in the broad He II $\lambda 4686$ emission line.

extragalactic emission-line objects (see MacAlpine et al. 1979). NGC 4593 is a type (R)SB(rs)b spiral galaxy at a redshift of $z=0.0090$.

\subsection{Spectroscopy}

MDM Observations. - We obtained spectra of the central region of NGC 4593 at 24 epochs between 2005 February and April with the Boller and Chivens CCD spectrograph on the $1.3 \mathrm{~m}$ telescope at MDM Observatory, at a spatial scale of 0.75 per pixel. For our campaign, we used a grating of 350 grooves $\mathrm{mm}^{-1}$, corresponding to a dispersion of $1.33 \AA$ pixel $^{-1}$. The spectral coverage was from $\sim 4300$ to $5700 \AA$, centered on $\mathrm{H} \beta \lambda 4861$ and the [O III] $\lambda \lambda 4959,5007$ lines, resulting in a spectral resolution of $7.6 \AA$ across this range. The slit width was set to 5.0 , projected on the sky, with a position angle of $90^{\circ}$. Figure 1 shows the mean and rms spectrum of NGC 4593, made from the complete set of MDM observations.

CrAO Observations. - We also acquired spectra of the nuclear region of NGC 4593 from the Nasmith spectrograph with the Astro-550 $580 \times 520$ pixel CCD (Berezin et al. 1991) on the $2.6 \mathrm{~m}$ Shajn telescope of the Crimean Astrophysical Observatory (CrAO). For these observations a 3."0 slit was used, with a $90^{\circ}$ position angle. Spectral wavelength coverage for this data set was from $\sim 4300$ to $5600 \AA$, with a dispersion of $2.0 \AA$ pixel $^{-1}$ and a spectral resolution of $8.2 \AA$.

\subsection{Photometry}

MAGNUMObservations.-In addition to spectral observations, we also obtained $V$-band photometry from the $2.0 \mathrm{~m}$ Multicolor Active Galactic Nuclei Monitoring (MAGNUM) Telescope at the Haleakala Observatories in Hawaii, imaged with the multicolor imaging photometer (MIP) as described by Kobayashi et al. (1998a, 1998b), Yoshii (2002), and Yoshii et al. (2003). Photometric reduction of NGC 4593 was similar to that described for other sources by Minezaki et al. (2004) and Suganuma et al.
(2006), except that the host-galaxy contribution to the flux within the aperture was not subtracted and the filter color term was not corrected, because these photometric data were later scaled to the MDM and CrAO continuum light curve (as described below). Also, minor corrections (of order $0.01 \mathrm{mag}$ or less) due to the seeing dependence of the host-galaxy flux were ignored.

\subsection{Light Curves}

Following reduction of the data, light curves were created for the subsequent cross-correlation analysis. The spectral fluxes of the MDM observations were calibrated based on the [O III] $\lambda 5007$ line flux between observed frame wavelengths of 5022-5062 $\AA$ in the mean spectrum. Individual spectra were scaled to this reference spectrum using software that employs a $\chi^{2}$ goodness of fit estimator (van Groningen \& Wanders 1992). Because few MDM observations were taken under photometric conditions, our absolute [O III] $\lambda 5007$ flux calibrations are not reliable. Therefore, the final light curves are calibrated to the $[\mathrm{O}$ III] $\lambda 5007$ flux given by Dietrich et al. (1994). Light curves from the CrAO spectra were made following a similar method; only the final light-curve flux measurements were not calibrated to the Dietrich et al. (1994) value because this data set was later scaled to the MDM data set, as described below. Continuum and line flux measurements were obtained by first fitting the continuum on either side of the $\mathrm{H} \beta$ line, between the wavelength ranges $4795-4815$ and $5120-5170 \AA$ in the observed frame. The $\mathrm{H} \beta$ flux was calculated by integrating above the continuum over the wavelength range 4825-4963 $\AA$. The continuum flux density was then taken to be the average over the range 5120-5170 $\AA$. Although at least some contamination from $\mathrm{Fe}$ II emission is unavoidable when measuring the continuum in the optical, we chose this continuum region because it is the cleanest window, with respect to this contamination, within the spectral coverage of our observations. In addition, Vestergaard \& Peterson (2005) demonstrate that, although Fe II emission is variable, the amplitude of variability is generally low. We do not expect Fe II contamination to significantly affect our measurements.

In order to intercalibrate the data from various sources (i.e., place them on a consistent flux scale), corrections to the measured line and continuum flux values are necessary because of systematic differences, mostly attributable to seeing and aperture effects (see Peterson et al. 1991). Given data sets from two sources, flux differences were determined by comparing all possible pairs of simultaneous observations between the two data sets. Here we relaxed the meaning of "simultaneous" to include pairs separated by at most 2.0 days to increase the number of pairs contributing to each comparison. However, this relaxed assumption does not produce significantly larger (i.e., less than $1.0 \%$ ) uncertainties, implying that there is no evidence for intrinsic variability on such short timescales. Based on the average difference in flux between these closely spaced pairs, a single multiplicative point-source correction factor, $\varphi_{\mathrm{CrAO}}=0.89$, was applied to all points in both the emission-line and continuum light curves of the secondary data set $(\mathrm{CrAO})$ to scale it to the primary set (MDM), following the methods of Peterson et al. (1991). Physically, this scaling factor accounts for differences in the amount of [O III] $\lambda 5007$ flux measured due to different aperture geometries and seeing between the two data sets. Seeing can also affect the internal calibrations of the continuum and $\mathrm{H} \beta$ emission-line flux measurements, based on $F([\mathrm{O}$ III $]$ 25007), which is assumed to be constant. However, given the large slit widths used in the current MDM and CrAO observations (5".0 and 3".0, respectively), as well as the compact (no larger than 1".7) emission region of [O III] in NGC 4593 (Schmitt et al. 2003), seeing is an 
TABLE 1

Continuum and H $\beta$ Fluxes for NGC 4593

\begin{tabular}{|c|c|c|c|}
\hline $\begin{array}{c}\text { JD } \\
(-2,450,000)\end{array}$ & $\begin{array}{c}F_{\lambda}(5100 \AA) \\
\left(10^{-15} \operatorname{ergs~s}^{-1} \mathrm{~cm}^{-2} \AA^{-1}\right)\end{array}$ & $\begin{array}{c}\mathrm{H} \beta \lambda 4861 \\
\left(10^{-13} \mathrm{ergs} \mathrm{s}^{-1} \mathrm{~cm}^{-2}\right)\end{array}$ & Data Set \\
\hline $3391.986 \ldots .$. & $10.69 \pm 0.10$ & $\ldots$ & MAGNUM \\
\hline 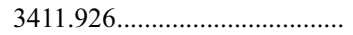 & $9.23 \pm 0.10$ & $\ldots$ & MAGNUM \\
\hline 3419.121....... & $9.86 \pm 0.05$ & $\ldots$ & MAGNUM \\
\hline 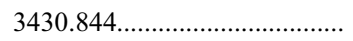 & $8.94 \pm 0.18$ & $4.59 \pm 0.09$ & MDM \\
\hline $3430.962 \ldots \ldots \ldots \ldots \ldots \ldots \ldots$ & $9.78 \pm 0.08$ & $\ldots$ & MAGNUM \\
\hline 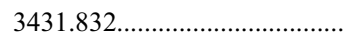 & $8.75 \pm 0.18$ & $4.52 \pm 0.09$ & MDM \\
\hline 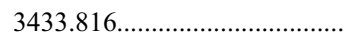 & $8.52 \pm 0.17$ & $4.61 \pm 0.09$ & MDM \\
\hline 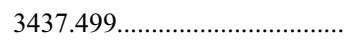 & $9.68 \pm 0.32$ & $4.67 \pm 0.10$ & $\mathrm{CrAO}$ \\
\hline 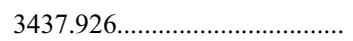 & $9.39 \pm 0.19$ & $4.77 \pm 0.10$ & MDM \\
\hline 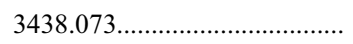 & $10.37 \pm 0.04$ & $\ldots$ & MAGNUM \\
\hline 3438.805 & $9.81 \pm 0.20$ & $5.03 \pm 0.10$ & MDM \\
\hline 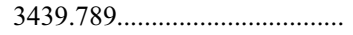 & $9.60 \pm 0.19$ & $5.00 \pm 0.10$ & MDM \\
\hline 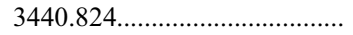 & $9.51 \pm 0.19$ & $5.08 \pm 0.10$ & MDM \\
\hline$\ldots$ & $9.24 \pm 0.19$ & $5.12 \pm 0.10$ & MDM \\
\hline 3442.820 & $8.92 \pm 0.18$ & $5.34 \pm 0.11$ & MDM \\
\hline 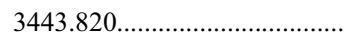 & $9.05 \pm 0.18$ & $5.23 \pm 0.11$ & MDM \\
\hline $3444.450 \ldots \ldots \ldots \ldots \ldots \ldots \ldots \ldots$ & $8.75 \pm 0.29$ & $5.08 \pm 0.11$ & $\mathrm{CrAO}$ \\
\hline 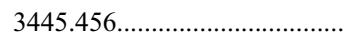 & $7.99 \pm 0.26$ & $5.07 \pm 0.11$ & $\mathrm{CrAO}$ \\
\hline 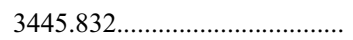 & $8.80 \pm 0.18$ & $5.01 \pm 0.10$ & MDM \\
\hline $3446.448 \ldots$. & $8.64 \pm 0.29$ & $4.91 \pm 0.10$ & $\mathrm{CrAO}$ \\
\hline 3446.816 & $8.63 \pm 0.17$ & $4.97 \pm 0.10$ & MDM \\
\hline $3450.832 \ldots \ldots$ & $8.19 \pm 0.16$ & $4.53 \pm 0.09$ & MDM \\
\hline 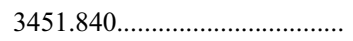 & $8.38 \pm 0.17$ & $4.36 \pm 0.09$ & MDM \\
\hline 3452.793 & $8.66 \pm 0.17$ & $4.27 \pm 0.09$ & MDM \\
\hline 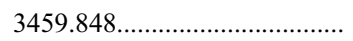 & $8.99 \pm 0.18$ & $4.56 \pm 0.09$ & MDM \\
\hline 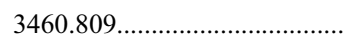 & $8.71 \pm 0.17$ & $4.58 \pm 0.09$ & MDM \\
\hline $3461.812 \ldots \ldots \ldots \ldots \ldots$ & $9.00 \pm 0.18$ & $4.56 \pm 0.09$ & MDM \\
\hline 3462.848 & $9.00 \pm 0.18$ & $4.63 \pm 0.09$ & MDM \\
\hline 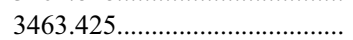 & $10.23 \pm 0.34$ & $4.78 \pm 0.10$ & $\mathrm{CrAO}^{\mathrm{a}}$ \\
\hline 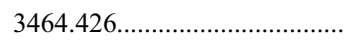 & $9.58 \pm 0.32$ & $4.76 \pm 0.10$ & $\mathrm{CrAO}^{\mathrm{a}}$ \\
\hline 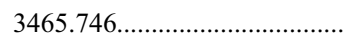 & $9.19 \pm 0.18$ & $4.80 \pm 0.10$ & MDM \\
\hline $3465.890 \ldots \ldots$ & $9.93 \pm 0.15$ & $\ldots$ & MAGNUM \\
\hline $3467.844 \ldots \ldots \ldots \ldots \ldots \ldots \ldots \ldots \ldots \ldots$ & $9.19 \pm 0.18$ & $4.70 \pm 0.09$ & MDM \\
\hline 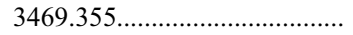 & $9.33 \pm 0.31$ & $4.87 \pm 0.10$ & $\mathrm{CrAO}$ \\
\hline $3469.785 \ldots \ldots \ldots \ldots$ & $9.41 \pm 0.19$ & $4.80 \pm 0.10$ & MDM \\
\hline 3470.364.............................. & $9.34 \pm 0.31$ & $5.05 \pm 0.11$ & $\mathrm{CrAO}$ \\
\hline 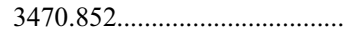 & $9.54 \pm 0.19$ & $5.00 \pm 0.10$ & MDM \\
\hline 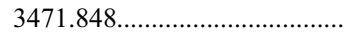 & $9.29 \pm 0.19$ & $4.90 \pm 0.10$ & MDM \\
\hline 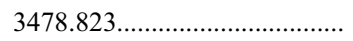 & $9.60 \pm 0.07$ & $\ldots$ & MAGNUM \\
\hline 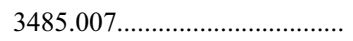 & $9.70 \pm 0.07$ & $\ldots$ & MAGNUM \\
\hline 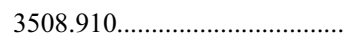 & $9.91 \pm 0.05$ & $\ldots$ & MAGNUM \\
\hline 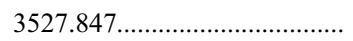 & $9.99 \pm 0.03$ & $\ldots$ & MAGNUM \\
\hline 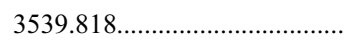 & $10.61 \pm 0.12$ & $\ldots$ & MAGNUM \\
\hline $3556.769 \ldots \ldots \ldots \ldots \ldots \ldots \ldots \ldots \ldots \ldots$ & $10.46 \pm 0.06$ & $\ldots$ & MAGNUM \\
\hline $3572.766 \ldots \ldots$ & $8.44 \pm 0.12$ & $\ldots$ & MAGNUM \\
\hline 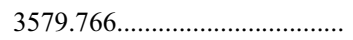 & $9.35 \pm 0.25$ & $\ldots$ & MAGNUM \\
\hline
\end{tabular}

${ }^{\text {a }}$ Continuum point omitted from the final light curve. See $\S 3$.

insignificant effect in our internal calibrations. Further tests of the effect of seeing on our internal flux calibrations are addressed in $\S 3$.

In addition to this multiplicative correction, an additive correction factor, $G_{\mathrm{CrAO}}=-1.60 \times 10^{-15} \mathrm{ergs} \mathrm{s}^{-1} \mathrm{~cm}^{-2} \AA^{-1}$, was also made to the continuum flux, accounting for the starlight contribution in the different apertures of the various data sets. These correction methods were employed to scale both the continuum and $\mathrm{H} \beta$ light curves from the $\mathrm{CrAO}$ observations to the MDM data, since the MDM data formed the largest individual set and thus served as the base on which to build the others. For the same reason, the photometric, $V$-band observations from the MAGNUM data were then scaled to the newly combined MDM and CrAO continuum light curve, with the additive correction factor $G_{\mathrm{MAGN}}=$ $0.65 \times 10^{-15} \mathrm{ergs} \mathrm{s}^{-1} \mathrm{~cm}^{-2} \AA^{-1}$. The continuum light curve was finally corrected for host-galaxy starlight, where for this slit geometry the contribution is measured to be $F_{\text {gal }}(5100 \AA)=$ $1.11_{-0.11}^{+0.10} \times 10^{-14} \mathrm{ergs} \mathrm{s}^{-1} \mathrm{~cm}^{-2} \AA^{-1}$ from a recent Hubble Space Telescope image taken with the Advanced Camera for Surveys High Resolution Camera. The methods by which this flux contribution was determined are described by Bentz et al. (2006a), and the complete results specific to this object will appear in a future paper. The full set of observations used in constructing these light curves can be found in Table 1, and Figure 2 shows the corresponding light curves.

\section{TIME SERIES ANALYSIS}

For the time series analysis, the light curves from Figure 2 were modified by first merging the three sets of observations by 


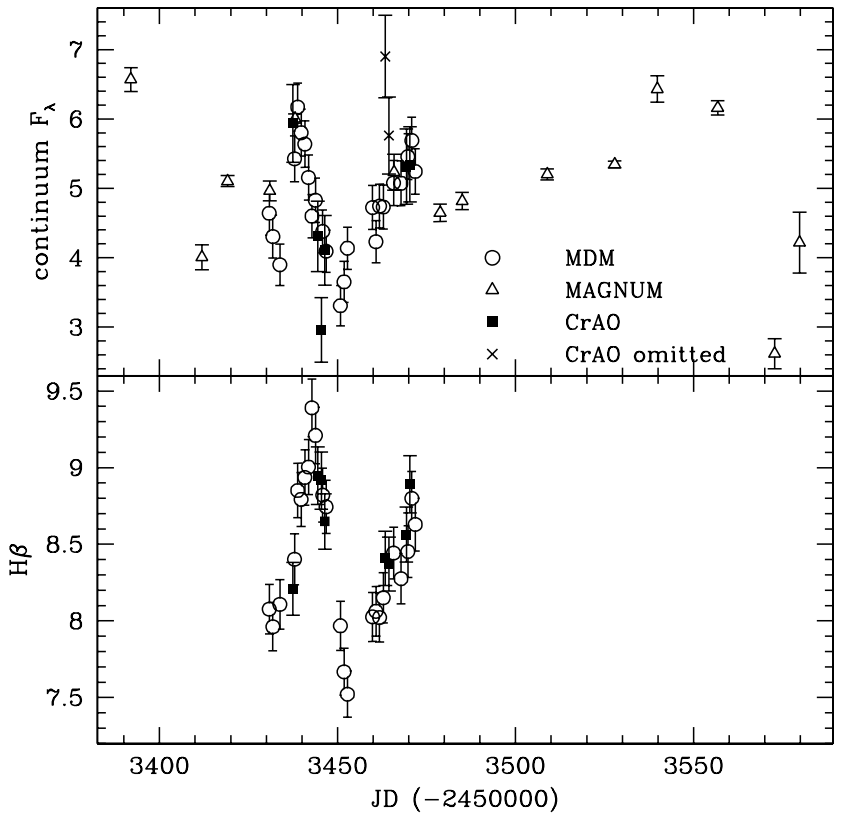

FIG. 2.-Light curve showing complete data sets from all three sources. Top, $5100 \AA$ continuum flux in units of $10^{-15} \mathrm{ergs} \mathrm{s}^{-1} \mathrm{~cm}^{-2} \AA^{-1}$; bottom, $\mathrm{H} \beta \lambda 4861$ line flux in units of $10^{-13} \mathrm{ergs} \mathrm{s}^{-1} \mathrm{~cm}^{-2}$. The crosses show the two continuum points that were later omitted from the final light curve (see $\S 3$ ).

binning data points more closely spaced than 0.5 day by using a variance-weighted average. Cross-correlation analysis was restricted to the subset of the light curves for which both continuum and emission-line measurements are available, i.e., JD 2,453,430-JD 2,453,472. In addition, two observations from the CrAO data set, JD 2,453,463.4 and JD 2,453,465.9, show anomalously high continuum flux, for reasons that could not be determined. Conducting the cross-correlation analysis including these points resulted in lag determinations consistent with removing the points, although the uncertainties in the lags were higher. Since this time period was otherwise well sampled, none of the other observations show a similar peak in continuum flux, and because the results from exclusion are consistent with inclusion, we omit these two continuum points from the final light curve.

Figure 3 shows the modified light curves that were used for the cross-correlation analysis. The statistical parameters describing these final light curves are shown in Table 2. Column (1) gives the spectral feature, and column (2) shows the number of data points in each light curve. Columns (3) and (4) list the mean and median sampling intervals, respectively, between data points. The mean flux with standard deviation is given in column (5), while column (6) shows the mean fractional error, based on comparison between closely spaced observations. Column (7) gives the excess variance, calculated as

$$
F_{\text {var }}=\frac{\sqrt{\sigma^{2}-\delta^{2}}}{\langle f\rangle},
$$

where $\sigma^{2}$ is the variance of the observed fluxes, $\delta^{2}$ is their mean square uncertainty, and $\langle f\rangle$ is the mean of the observed fluxes. Finally, column (8) is the ratio of the maximum to minimum flux in the light curves.

We conducted the time series analysis by cross-correlating the continuum light curve with the $\mathrm{H} \beta$ light curve using two methods designed for unevenly spaced observations. The primary method

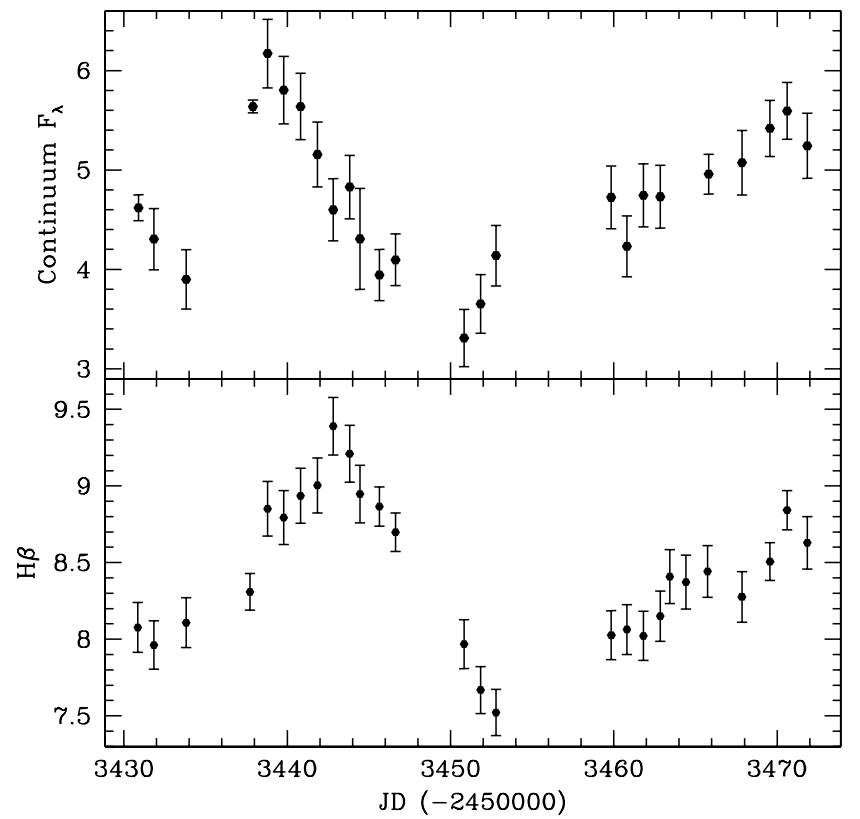

FIG. 3.- Light curve showing subset of data constrained to the time frame of the MDM observations used for time series analysis. Observations within 0.5 day of each other have been averaged together. Top, Continuum at $5100 \AA$ in units of $10^{-15} \mathrm{ergs} \mathrm{s}^{-1} \mathrm{~cm}^{-2} \AA^{-1}$; bottom, $\mathrm{H} \beta \lambda 4861$ line flux in units of $10^{-13} \mathrm{ergs} \mathrm{s}^{-1} \mathrm{~cm}^{-2}$.

uses an interpolation scheme that averages two results: first, crosscorrelating an interpolated continuum light curve with the original emission-line light curve and, second, cross-correlating the original continuum with an interpolated emission-line light curve (Gaskell \& Peterson 1987). We employed an interpolation interval of 0.5 day (equal to half of the median time span between observations), chosen to create equal spacing on a scale smaller than that of the actual data, yet large enough to prevent the introduction of artifacts due to the correlation of adjacent interpolated flux values. The correlation coefficient, $r$, is computed from pairs of points from each light curve matched by imposing different lags on the trailing curve. For a given time lag realization, unpaired points at the beginning or end of the time series are excluded from the correlation analysis. By calculating $r$ for multiple potential lag times, a cross-correlation function (CCF) is constructed, shown in Figure 4, which gives the correlation coefficient at each of these different lag times, $\tau$. From the CCF, we can characterize the time delay through two parameters. The peak lag, $\tau_{\text {peak }}$, is simply taken as the lag time that produces the highest correlation coefficient, $r_{\max }$, whereas the centroid lag, $\tau_{\text {cent }}$, is the centroid of the CCF, computed from values with $r \geq 0.8 r_{\max }$.

The uncertainties in the time lag measurements were computed via model-independent Monte Carlo simulations and based on the bootstrap method referred to as FR/RSS (for flux redistribution/ random subset selection) and described by Peterson et al. (1998), which uses the modifications of Peterson et al. (2004). A large number of realizations of this method (e.g., 10,000 in this study) are employed to build up two distributions of CCFs: the crosscorrelation centroid distribution (CCCD) and the cross-correlation peak distribution (CCPD). The values $\tau_{\text {cent }}$ and $\tau_{\text {peak }}$ are the means of these respective distributions. The uncertainties are calculated such that $15.87 \%$ of the realizations yield values larger than the mean plus the upper error, and $15.87 \%$ yield values smaller than the mean minus the lower error; these would correspond to the $1 \sigma$ errors in the case of a Gaussian distribution. Table 3 gives the 
TABLE 2

Light-Curve Statistics

\begin{tabular}{|c|c|c|c|c|c|c|c|}
\hline \multirow[b]{2}{*}{$\begin{array}{l}\text { TIME SERIES } \\
\text { (1) }\end{array}$} & \multirow[b]{2}{*}{$\begin{array}{l}N \\
(2)\end{array}$} & \multicolumn{2}{|c|}{ SAMPLING INTERVAL (days) } & \multirow[b]{2}{*}{$\begin{array}{l}\text { Mean Flux }{ }^{a} \\
\text { (5) }\end{array}$} & \multirow[b]{2}{*}{$\begin{array}{c}\text { Mean Fractional ERror } \\
(6)\end{array}$} & \multirow[b]{2}{*}{$\begin{array}{l}F_{\text {var }} \\
(7)\end{array}$} & \multirow[b]{2}{*}{$\begin{array}{c}R_{\max } \\
(8)\end{array}$} \\
\hline & & $\begin{array}{l}\langle T\rangle \\
(3)\end{array}$ & $\begin{array}{l}T_{\text {median }} \\
\text { (4) }\end{array}$ & & & & \\
\hline ....................... & 25 & 1.7 & 1.0 & $4.8 \pm 0.7$ & 0.06 & 0.14 & $1.87 \pm 0.19$ \\
\hline $\mathrm{H} \beta$ & 27 & 1.6 & 1.0 & $8.4 \pm 0.5$ & 0.02 & 0.05 & $1.25 \pm 0.04$ \\
\hline
\end{tabular}

${ }^{\text {a }}$ Same flux units as in Table 1 for $5100 \AA$ continuum and $\mathrm{H} \beta$, respectively.

calculated values of $\tau_{\text {cent }}$ and $\tau_{\text {peak }}$ for NGC 4593 after being corrected for time dilation.

The second cross-correlation method we employ creates a discrete correlation function (DCF), described by Edelson \& Krolik (1988) and including modifications by White \& Peterson (1994). Shown in Figure 4, the DCF is created by determining correlations at different lag times between the $\mathrm{H} \beta$ and continuum light curves, just as in the interpolation method, but through time binning of data, rather than interpolation. Undersampled data or data with large time gaps could lead to spurious lag determinations by using the interpolation method, in which case there are advantages to using the DCF method. For example, only the actual data are used in the analysis, and, due to the binning, statistical uncertainties can be assigned to the correlation coefficient for each bin (see White \& Peterson 1994). For this analysis, a time bin of 2.0 days was adopted. One can see from Figure 4 that the DCF agrees well with the CCF from the interpolation method, showing that interpolation is not introducing artifacts. Figure 4 also shows the autocorrelation function (ACF), which is computed by cross-correlating the continuum with itself. The $\mathrm{ACF}$ is interesting because the CCF is the convolution of this distribution (ACF) and the transfer function, which describes the emission-line response to the continuum variations and is the quantity the reverberation mapping is fundamentally attempting to recover (see Peterson 1993).

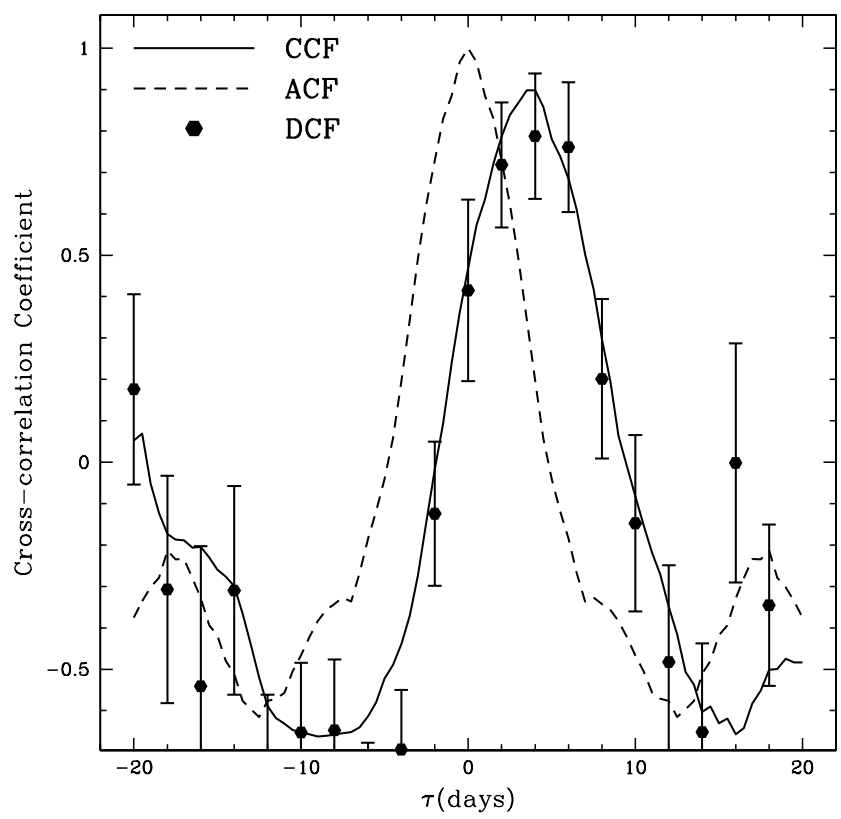

FIG. 4.-Cross-correlation function (CCF), discrete correlation function (DCF), and auto correlation function (ACF) from time series analysis of the continuum and $\mathrm{H} \beta$ light curves of NGC 4593 .
The CCFs in Figure 4 also demonstrate that our continuum and $\mathrm{H} \beta$ emission-line internal flux calibration to the $[\mathrm{O}$ III $] \lambda 5007$ emission line are not adversely affected by nightly changes in seeing. Because calibration errors due to seeing would result in correlated errors, with both the continuum and the line flux affected in the same direction, if variable seeing is a significant effect, it would lead to a spike in the CCF at a lag of 0 days. Figure 4 clearly shows no such feature in either the CCF or DCF for NGC 4593. Bentz et al. (2006b) report on results for NGC 4151 from this same observing campaign and do not see any evidence for this zero-lag spike, either. The only object from this campaign in which a zero-lag spike is detected in the CCF is NGC 5548 (Bentz et al. 2006c), which was in a very low flux state at the time. In this case, the amplitude of variability was so low that the weak correlated-error signature was above the detection threshold.

To investigate the possibility of detecting bulk motions within the BLR, we then broke the $\mathrm{H} \beta$ broad emission line into velocity bins and performed the cross-correlation anaylsis between these individual bins. First, the $\mathrm{H} \beta$ line was divided in half, based on the peak in the mean spectrum (see Fig. 1), with the blue side defined by the wavelengths $4825-4903 \AA$ and the red side defined by the wavelengths $4903-4963 \AA$. The flux was determined by integrating the line flux above the same continuum fit as described above. The lags determined through this cross-correlation analysis are $\tau_{\text {cent }}=0.5 \pm 1.0$ day and $\tau_{\text {peak }}=0.7_{-1.5}^{+1.0}$ day, with variations in the blue side slightly lagging behind those in the red side. Second, the line was broken into a "core" and a "wing" component, such that the integrated flux in the core was equal to the summed flux in both red and blue wings. These regions were defined from the mean spectrum such that the blue wing constituted the integrated flux between the wavelengths 4825 and $4881.8 \AA$, the core between 4881.8 and $4924.2 \AA$, and the red wing between 4924.2 and $4963 \AA$. The lags determined for this scenario are $\tau_{\text {cent }}=0.5 \pm 0.7$ day and $\tau_{\text {peak }}=0.1 \pm 1.0$ day, with variations in the wings leading the core, as would be expected. The CCFs from both of these analyses are shown in Figure 5. Unfortunately, any signature of inflow/outflow or bulk Keplarian motions was too weak to be detected with confidence

TABLE 3

Reverberation Results

\begin{tabular}{|c|c|}
\hline Parameter & Value \\
\hline 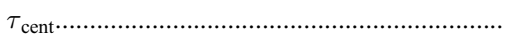 & $3.73 \pm 0.75$ days \\
\hline$\tau_{\text {peak }} \cdots \ldots \ldots \ldots$ & $3.4_{-1.0}^{+0.5}$ days \\
\hline$\sigma_{\text {line }}($ mean $) \ldots \ldots$ & $1790 \pm 3 \mathrm{~km} \mathrm{~s}^{-1}$ \\
\hline FWHM (mean) & $5143 \pm 16 \mathrm{~km} \mathrm{~s}^{-1}$ \\
\hline$\sigma_{\text {line }}(\mathrm{rms})$ & $1561 \pm 55 \mathrm{~km} \mathrm{~s}^{-1}$ \\
\hline 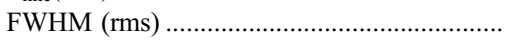 & $4141 \pm 416 \mathrm{~km} \mathrm{~s}^{-1}$ \\
\hline$M_{\mathrm{BH}}$ & $(9.8 \pm 2.1) \times 10^{6} M_{6}$ \\
\hline
\end{tabular}




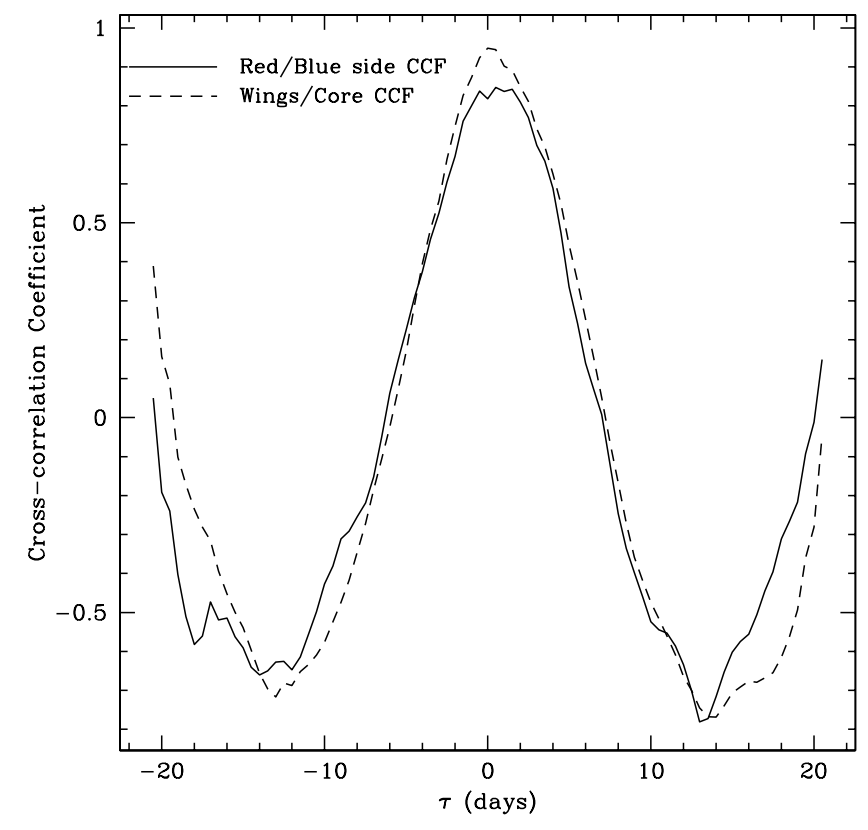

FIG. 5.-Cross-correlation functions (CCF) from time series analysis of the red and blue sides of the broad $\mathrm{H} \beta$ emission line (solid line) and of the wings and the core of the $\mathrm{H} \beta$ emission line (dashed line) of NGC 4593.

by this investigation, as the lags measured for both scenarios were consistent with zero.

\section{BLACK HOLE MASS}

Assuming that the motions within the line-emitting region are gravitationally dominated so that the virial theorem applies, the mass of the black hole can be defined such that

$$
M_{\mathrm{BH}}=\frac{f_{c} \tau(\Delta V)^{2}}{G},
$$

where $\tau$ is the measured emission-line time delay and $\Delta V$ is the emission-line width, which can be characterized by either the full width at half-maximum (FWHM) of the broad emission line or the emission-line dispersion, $\sigma_{\text {line. }}$ The dimensionless factor $f$ depends on the structure, kinematics, and inclination of the BLR and is of order unity. By normalizing the reverberation-based black hole masses to the $M_{\mathrm{BH}} \sigma_{\star}$ relationship for quiescent galaxies, Onken et al. (2004) have shown that $f$ has an average value of 5.5 when the emission-line dispersion, or second moment of the profile, is used (as opposed to the $\mathrm{FWHM}^{11}$ ) for $\Delta V$.

After first removing the narrow-line component of the $\mathrm{H} \beta$ emission line, the FWHM and the line dispersion were measured from both the mean and the rms spectra of NGC 4593. Peterson et al. (2004) describe in detail the bootstrap method used to determine these quantities and their respective uncertainties. The measured values for the FWHM and $\sigma_{\text {line }}$ are given in Table 3 .

To calculate the black hole mass for NGC 4593, we use the centroid lag, $\tau_{\text {cent }}$, for the time delay, $\tau$, and the line dispersion, $\sigma_{\text {line }}$, of the $\mathrm{H} \beta$ emission line, measured from the rms spectrum, for the emission-line width, $\Delta V$. Peterson et al. (2004) argue that this combination of parameters gives the most reliable black hole mass determinations, based on virial arguments and fits between

\footnotetext{
11 See Collin et al. (2006) for a discussion of systematic differences between the use of the FWHM and the line dispersion in calculating the virial product and thus $M_{\mathrm{BH}}$.
}

all possible combinations of parameters. The black hole mass calculated for NGC 4593 from this work is $M_{\mathrm{BH}}=(9.8 \pm 2.1) \times$ $10^{6} M_{\odot}$, where the uncertainties quoted include statistical and observational considerations, but not an intrinsic uncerainty in the method, no larger than a factor of $2-3$, which accounts for unknowns such as inclination.

Our result is consistent with the mass estimate based on a previous reverberation program. Onken et al. (2003) reanalyzed observations of NGC 4593 by the Lovers of Active Galaxies (LAG) consortium (Dietrich et al. 1994) and determined a black hole mass based on $\mathrm{H} \beta$ to be $M_{\mathrm{BH}}=5.4_{-7.0}^{+9.4} \times 10^{6} M_{\odot}$, where we have used the Onken et al. (2004) calibration (Peterson et al. 2004). The improved time sampling of the new reverberationmapping observations has significantly decreased the uncertainties in the black hole mass estimate for this object.

\section{DISCUSSION AND CONCLUSION}

Results presented here for the emission-line lag and black hole mass of NGC 4593 of $\tau_{\text {cent }}=3.73 \pm 0.75$ days and $M_{\mathrm{BH}}=$ $(9.8 \pm 2.1) \times 10^{6} M_{\odot}$ for these respective quantities represent a factor-of-several improvement over past measurements. Previous measurements for this object from LAG data exhibited much larger uncertainties, due in part to the average sampling interval, which, due mostly to bad weather, was much larger (by about a factor of 4) than the emission-line lag of this object. In addition, for rather short observing campaigns, some of the success of reverberation mapping comes from serendipitously observing a large-variability event during the campaign. The best results come from observing a large increase and then decrease (or vice versa) in the flux, which we observed in this campaign, but was unfortunately not seen to the same degree in the previous campaign of NGC 4593. This represents yet another example of the need for longer observing campaigns.

The results reported here were obtained as part of a larger spectroscopic monitoring campaign whose primary goal was to improve the emission-line lag and black hole mass measurements for some of the nearest, apparently brightest, AGNs. The proximity of these AGNs makes them especially important not only as calibrators of the AGN $M_{\mathrm{BH}^{-}} \sigma_{*}$ and BLR radius-luminosity relationships (Bentz et al. 2006a), but also as candidates for measurement of their black hole masses through other means that depend on high angular resolution. Key elements of this program were (1) scheduled daily observations of each of these relatively low-luminosity AGNs, (2) supporting observations at multiple sites to mitigate the effects of gaps in the time series caused by weather, and (3) high-quality homogeneous data that permitted relative spectrophotometric flux calibration at better than the $2 \%$ level. Our 42 night program yielded improved $\mathrm{H} \beta$ lags and black hole masses, with a factor-of-several improvement in precision relative to previous campaigns for three of the six AGNs in our sample, NGC 4151 (Bentz et al. 2006b), NGC 5548, which was observed in the lowest luminosity state yet recorded (Bentz et al. 2006), and NGC 4593, as described here. Three other AGNs in our program, NGC 3227, NGC 3516, and NGC 4051, were insufficiently variable during this relatively brief campaign, underscoring the point that programs of longer duration are necessary for detection of variability signatures favorable for reverberation analysis.

We acknowledge support for this work from the National Science Foundation through grant AST 02-05964 to Ohio State University and the Civilian Research and Development Foundation 
through grant UP1-2549-CR-03. K. D. D. is supported by a GK-12 Fellowship from the National Science Foundation. M. C. B. is supported by a Graduate Fellowship from the National Science Foundation. E. M. C. gratefully acknowledges support from PPARC. M. V. acknowledges financial support from NSF grant
AST 03-07384 to the University of Arizona. This research has made use of the NASA/IPAC Extragalactic Database (NED), which is operated by the Jet Propulsion Laboratory, California Institute of Technology, under contract with the National Aeronautics and Space Administration.

REFERENCES

Bentz, M. C., Peterson, B. M., Pogge, R. W., Vestergaard, M., \& Onken, C. A 2006a, ApJ, 644, 133

Bentz, M. C., et al. 2006b, ApJ, 651, 775

2006c, ApJ, submitted

Berezin, V. Y., et al. 1991, Soviet Astron. Lett., 17, 405

Blandford, R. D., \& McKee, C. F. 1982, ApJ, 255, 419

Collin, S., Kawaguchi, T., Peterson, B. M., \& Vestergaard, M. 2006, A\&A, 456, 75

Dietrich, M., et al. 1994, A\&A, 284, 33

Edelson, R. A., \& Krolik, J. H. 1988, ApJ, 333, 646

Gaskell, C. M., \& Peterson, B. M. 1987, ApJS, 65, 1

Horne, K., Peterson, B. M., Collier, S. J., \& Netzer, H. 2004, PASP, 116, 465

Kobayashi, Y., Yoshii, Y., Peterson, B. A., Minezaki, T., Enya, K., Suganuma, M., \& Yamamuro, T. 1998a, Proc. SPIE, 3354, 769

Kobayashi, Y., et al. 1998b, Proc. SPIE, 3352, 120

Lewis, D. W., MacAlpine, G. M., \& Koski, A. T. 1978, BAAS, 10, 388

MacAlpine, G. M., Williams, G. A., \& Lewis, D. W. 1979, PASP, 91, 746

Minezaki, T., Yoshii, Y., Kobayashi, Y., Enya, K., Suganuma, M., Tomita, H., Aoki, T., \& Peterson, B. A. 2004, ApJ, 600, L35
Onken, C. A., Ferrarese, L., Merritt, D., Peterson, B. M., Pogge, R. W., Vestergaard, M., \& Wandel, A. 2004, ApJ, 615, 645

Onken, C. A., Peterson, B. M., Dietrich, M., Robinson, A., \& Salamanca, I. M. 2003, ApJ, 585, 121

Peterson, B. M. 1993, PASP, 105, 247

Peterson, B. M., Wanders, I., Bertram, R., Hunley, J. F., Pogge, R. W., \& Wagner, R. M. 1998, ApJ, 501, 82

Peterson, B. M., et al. 1991, ApJ, 368, 119 2004, ApJ, 613, 682

Schmitt, H. R., Donley, J. L., Antonucci, R. R. J., Hutchings, J. B., \& Kinney, A. L. 2003, ApJS, 148, 327

Suganuma, M., et al. 2006, ApJ, 639, 46

van Groningen, E., \& Wanders, I. 1992, PASP, 104, 700

Vestergaard, M., \& Peterson, B. M. 2005, ApJ, 625, 688

White, R. J., \& Peterson, B. M. 1994, PASP, 106, 879

Yoshii, Y. 2002, in New Trends in Theoretical and Observational Cosmology, ed. K. Sato \& T. Shiromizu (Tokyo: Universal Academy), 235

Yoshii, Y., Kobayashi, Y., \& Minezaki, T. 2003, BAAS, 202, 3803 